\title{
Distance-Intensity for Image Registration
}

\author{
Rui Gan and Albert C. S. Chung \\ Lo Kwee-Seong Medical Image Analysis Laboratory, \\ Department of Computer Science, \\ The Hong Kong University of Science and Technology, Hong Kong \\ \{raygan, achung\}@cs.ust.hk
}

\begin{abstract}
In this paper, a novel one-element voxel attribute, namely distance-intensity (DI), is defined for associating spatial information with image intensity for registration tasks. For each voxel in an image, the DI feature encodes spatial information at a global level, and is about the distance of the voxel to its closest object boundary, together with the original intensity information. Without the help of image segmentations, the computation of the DI map is carried out by applying a Poisson process on a vector field that combines both gradient and distance-gradient. Mutual information (MI) is adopted as a similarity measure on the DI feature space. A multi-resolution registration method is then used for aligning multi-modal images. Experimental results show that, as compared with the conventional MI-based method, the proposed method has longer capture ranges at different image resolutions. This leads to more robust registrations. Randomized registration experiments on clinical 3D CT, MR-T1 and MR-T2 datasets demonstrate that the new method gives higher success rates than the traditional MI-based method.
\end{abstract}

\section{Introduction}

General promising results have shown that mutual information (MI) as a voxel intensity-based similarity measure is well-suited for multi-modal image registration [12]. However, it has been suggested that the conventional MI-based registration can result in misalignment for some cases 34 and then room for improvement exists. The standard MI measure only takes intensity information into account. Therefore, a known disadvantage is the lack of concern on any spatial information (neither local nor global) which may be present in individual images to be registered [56]. As a simple illustration, a random perturbation of image points identically on both images results in unchanged MI value as that of the original images.

Several researchers have proposed adaptations of the standard MI-based registration framework to incorporate spatial information. Pluim et al. 4 multiplies the conventional MI measure with an external local gradient term to ensure the alignment of locations of tissue transitions. The probing results indicated that the registration function of the combined measure is smoother than that of the standard MI measure. But this approach does not directly extend the MI based similarity measure. Butz et al. [7] applies MI to edge measure (e.g., gradient 
magnitude) space to align object surfaces. However, MI based on edge measure is sensitive to the sparseness of joint edge feature histograms. This may increase the difficulty of the optimization procedure. Moreover, Rueckert et al. [6] exploits higher-order MI for 4D joint histograms which are built on the co-occurrence of intensity pairs of adjacent points. This method was shown to be robust to local intensity variation. However, only one neighbor is considered at a time in this approach and plenty of spatial information which may be present globally or within large neighborhood system has been ignored.

In this paper, a novel one-element voxel attribute, namely distance-intensity (DI), is defined to incorporate spatial information with intensity for registration tasks. The DI feature encodes globally defined spatial information for each voxel. This is about the distance of the voxel to its closest object boundary, together with original intensity information. Without the help of image segmentations, the computation of DI map is carried out by applying a Poisson process on a vector field that combines both gradient and distance-gradient. Then, mutual information is exploited as a similarity measure on the DI feature space. To increase computational efficiency and robustness of the proposed method, the registration procedure is a multi-resolution iterative process.

Based on the results on clinical 3D CT, MR-T1 and MR-T2 image volumes, it is experimentally shown that the proposed method has relatively longer capture ranges 11 than the traditional MI-based method at different image resolutions. This can obviously make the multi-resolution image registration more robust. Moreover, the results of around 400 randomized registration experiments reveal that our method gives higher success registration rates than the conventional MI-based method.

\section{Distance-Intensity Attribute}

\subsection{Definition}

In our proposed registration approach, a novel one-element attribute, namely distance-intensity (DI), is assigned to each voxel in an image. Within individual images, the DI feature is designed for consolidating spatial information at a global level with intensity. In other words, the DI feature depends not only on image intensity, but also on the distance of a voxel to its closest object boundary.

Given an image $I(\mathbf{v})$ over domain $\Omega$, where $\mathbf{v}=(x, y, z)$ denotes voxel position, we define a distance-intensity (DI) map, $D I(\mathbf{v})$, of the image as

$$
D I(\mathbf{v})=I(\mathbf{v})+(I(\mathbf{v})-I(\mathbf{v}+\mathbf{d}(\mathbf{v}))) \log _{D}|\mathbf{d}(\mathbf{v})|,
$$

where $\mathbf{d}(\mathbf{v})$ is the vector from $\mathbf{v}$ to the closest boundary voxel of other object 2 and $D=\max _{\mathbf{v}}|\mathbf{d}(\mathbf{v})|$. Here the function $\log _{D}(\cdot)$ limits the influence of $\mathbf{d}(\mathbf{v})$.

${ }^{1}$ Capture range represents the range of alignments from which a registration algorithm can converge to the correct maximum.

2 This implies that two voxels $\mathbf{v}$ and $\mathbf{v}+\mathbf{d}(\mathbf{v})$ belong to different objects. 
Following this definition, when a voxel is at or near object boundary, the DI value approximates its original intensity value. Thus, structure transitions remain unchanged. On the other hand, when voxel position moves from boundaries towards interiors of homogenous regions (either background regions or anatomical structures), the DI value smoothly and gradually varies. With this property, the DI map of homogenous regions can provide global and detailed spatial information about the distance of a voxel to its closest object boundary, as well as intensity information. A graphical illustration for these properties will be presented in Section 2.3 .

\subsection{Computation}

It is noted that there is no available object segmentation (or boundary) in our registration process, i.e., $\mathbf{d}(\mathbf{v})$ is not derivable. However, we found that the gradient of DI map can be robustly estimated. Consequently, an accurate and smooth solution of the DI map can be computed by applying a Poisson process on a vector field. The estimated solution approximates the gradient of DI map.

The gradient of DI map, $\nabla D I(\mathbf{v})$, is given as follows,

$$
\begin{aligned}
\nabla D I(\mathbf{v}) & =\nabla I(\mathbf{v})+(I(\mathbf{v})-I(\mathbf{v}+\mathbf{d}(\mathbf{v}))) \frac{\nabla|\mathbf{d}(\mathbf{v})|}{|\mathbf{d}(\mathbf{v})| \log D} \\
& +\left(\nabla I(\mathbf{v})-\nabla I(\mathbf{v}+\mathbf{d}(\mathbf{v})) \cdot \frac{\partial(\mathbf{v}+\mathbf{d}(\mathbf{v}))}{\partial \mathbf{v}}\right) \log _{D}|\mathbf{d}(\mathbf{v})|
\end{aligned}
$$

where $\frac{\partial(\mathbf{v}+\mathbf{d}(\mathbf{v}))}{\partial \mathbf{v}}$ is the Jacobian matrix. Note that as compared with the first two terms in Eq. 2, the third term provides little influence: If $\mathbf{v}$ is inside homogenous regions, $\nabla I(\mathbf{v})$ and $\nabla I(\mathbf{v}+\mathbf{d}(\mathbf{v}))$ tend to zero; otherwise, when $\mathbf{v}$ is at or close to boundary, $\log _{D}|\mathbf{d}(\mathbf{v})|$ is tiny and inclines to zero. Therefore, we have

$$
\nabla D I(\mathbf{v}) \approx \nabla I(\mathbf{v})+(I(\mathbf{v})-I(\mathbf{v}+\mathbf{d}(\mathbf{v}))) \frac{\nabla|\mathbf{d}(\mathbf{v})|}{|\mathbf{d}(\mathbf{v})| \log D}
$$

This represents a weighted combination of gradient and distance-gradient.

Distance-Gradient: The distance-gradient operator $\left(\nabla_{d}\right)$ on two different voxels $\left(\mathbf{v}_{1}\right.$ and $\left.\mathbf{v}_{2}\right)$ is defined as

$$
\nabla_{d} I\left(\mathbf{v}_{1}, \mathbf{v}_{2}\right)=\left(I\left(\mathbf{v}_{1}\right)-I\left(\mathbf{v}_{2}\right)\right) \frac{\mathbf{v}_{1}-\mathbf{v}_{2}}{\left|\mathbf{v}_{1}-\mathbf{v}_{2}\right|^{2}}
$$

With this definition, the second term in Eq. 3 becomes $\frac{1}{\log D} \nabla_{d} I(\mathbf{v}, \mathbf{v}+\mathbf{d}(\mathbf{v}))$ by the fact that $\nabla|\mathbf{d}(\mathbf{v})|=\frac{\mathbf{d}(\mathbf{v})}{|\mathbf{d}(\mathbf{v})|}$ almost everywhere. Moveover, we make the following hypothesis, which is often satisfied in practice,

$$
\left|\nabla_{d} I(\mathbf{v}, \mathbf{v}+\mathbf{d}(\mathbf{v}))\right|=\max _{\mathbf{v}^{\prime} \in \Omega}\left|\nabla_{d} I\left(\mathbf{v}, \mathbf{v}^{\prime}\right)\right| .
$$


Consequently, Eq. 3 becomes

$$
\begin{array}{r}
\nabla D I(\mathbf{v}) \approx \mathbf{F}(\mathbf{v})=\nabla I(\mathbf{v})+\frac{1}{\log D} \nabla_{d} I(\mathbf{v}, \hat{\mathbf{v}}), \\
\text { with } \hat{\mathbf{v}}=\arg \max _{\mathbf{v}^{\prime} \in \Omega}\left|\nabla_{d} I\left(\mathbf{v}, \mathbf{v}^{\prime}\right)\right| .
\end{array}
$$

Poisson Process: Following Eq. 6, the vector field $\mathbf{F}(\mathbf{v})$ approximates $\nabla D I(\mathbf{v})$. In order to compute the DI map, one may use a direct integral approach on $\mathbf{F}(\mathbf{v})$. However, we have observed that it is unstable for real applications due to the insufficient capability of handling noise. The noise is cumulated and may result in a quite noticeable error. Therefore, we propose to minimize the following energy functional to derive an optimal solution of DI map, $\widehat{D I}(\mathbf{v})$,

$$
\iiint_{\mathbf{v} \in \Omega}|\nabla \widehat{D I}(\mathbf{v}(x, y, z))-\mathbf{F}(\mathbf{v}(x, y, z))|^{2} d x d y d z .
$$

$\widehat{D I}(\mathbf{v})$ can be obtained by solving a Poisson equation [8], $\nabla^{2} \widehat{D I}=\nabla \cdot \mathbf{F}$, where $\nabla^{2}=\left(\frac{\partial^{2}}{\partial x^{2}}+\frac{\partial^{2}}{\partial y^{2}}+\frac{\partial^{2}}{\partial z^{2}}\right)$ and $\nabla \cdot$ are Laplacian and divergence operators respectively. Obtaining the unique solution of Poisson equation is a well studied problem. In our work, Neumann boundary conditions are exploited. Then, the gradient descent flow minimizing the total energy is given by

$$
\widehat{D I}_{t}(\mathbf{v}(x, y, z))=\nabla^{2} \widehat{D I}(\mathbf{v}(x, y, z))-\nabla \cdot \mathbf{F}(\mathbf{v}(x, y, z)) .
$$

\subsection{Graphical Illustration}

As a detailed description, we computed the DI map of a clinical CT image volume obtained from the Retrospective Image Registration Evaluation (RIRE) project3. A slice from the volume is shown in Fig. 11, while Fig. 15 presents the corresponding slice from the DI map. (Note that values from individual images are re-scaled to $[0,1]$ for a fair comparison.) It is observed that, for those voxels at or near object boundaries, their DI values approximate the original intensity values. This implies that structure transitions remain unchanged. Meanwhile, when voxel position moves from boundaries towards interiors of homogenous regions (either background regions or anatomical structures), the DI value smoothly and gradually varies. However, due to the limitation of image quality, such smooth changes may not be clearly displayed in Fig. 11.

Furthermore, Figs. 1b and 1 respectively present the value profiles of the same line (marked as red dashed lines) in Figs. 17a and 1 1k. As suggested by Fig. $1 \mathrm{~d}$, the value variation from boundaries towards homogenous regions is smooth and gradual. It is worth noting that, although there is little intensity change at

\footnotetext{
${ }^{3}$ The images and the standard transformation(s) were provided as part of the project, Retrospective Image Registration Evaluation, National Institutes of Health, Project Number 8R01EB002124-03, Principal Investigator, J. Michael Fitzpatrick, Vanderbilt University, Nashville, TN.
} 


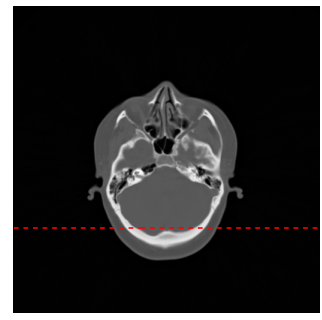

(a) A CT slice

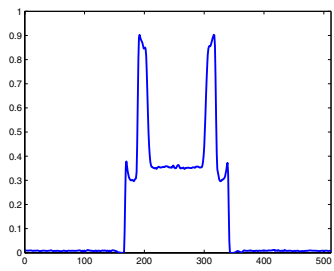

(b) Value profile

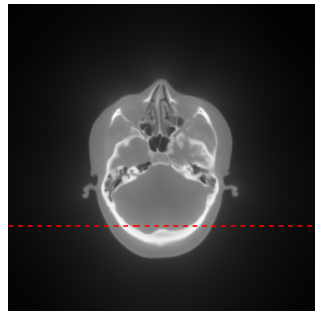

(c) DI map

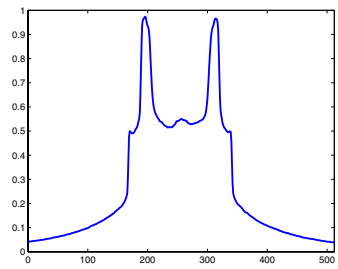

(d) Value profile

Fig. 1. (a) and (c) are slices respectively selected from a clinical CT image volume and its corresponding DI map. (b) and (d) are value profiles of lines in (a) and (c), which are marked as red dashed lines.

the middle of the line in Fig. 1b, a small and smooth saddle can be found in Fig. 1fd located at the corresponding position. The raised white boundary slightly below the line cause this saddle. It is because, as discussed above, the DI feature encodes spatial information at a global level.

\section{Mutual Information (MI) Based Image Registration}

As we have discussed above, the DI feature encodes spatial information at a global level together with original intensity information. We adopt it as voxel attribute for registration tasks. Mutual information (MI) 9] is then exploited as a similarity measure to measure the degree of dependence of the DI feature space. Given a geometric transformation, the 2D joint DI distribution can be approximated by either Parzen windowing or histogramming [10. Histogramming is employed in this paper because the approach is computationally efficient. The trilinear partial volume distribution interpolation [1] is exploited to update the joint histogram for non-grid alignment.

To accelerate the registration process and ensure the robustness of the proposed method, we exploit a multi-resolution approach based on the Gaussian Pyramid representation 2. Rough estimates can be found using downsampled images and treated as starting values for optimization at higher resolutions. Then 
the fine-tuning of the solution can be derived at the original image resolution. In this paper, the MI value at each resolution is maximized via the Powell's direction set method in multidimensions [1].

\section{Experimental Results and Discussions}

To evaluate the MI similarity measure on the novel distance-intensity attribute (hereafter referred to as MI-DI) and the proposed multi-resolution registration method, we have performed experiments on different image modalities: CT, rectified MR-T1 (T1-rec), and rectified MR-T2 (T2-rec). The traditional MI similarity measure on intensity (hereafter referred to as MI-I) [1|2] has also been applied for comparison.

\subsection{Comparisons on Capture Range}

CT - T1 (3D - 3D) Registration. Three pairs of clinical CT (around $512 \times 512 \times 30$ voxels and $\left.0.65 \times 0.65 \times 4 \mathrm{~mm}^{3}\right)$ and T1-rec $(256 \times 256 \times 26$ voxels and around $1.26 \times 1.26 \times 4.1 \mathrm{~mm}^{3}$ ) image volumes - datasets \#1, \#2 and \#3were obtained from RIRE. Note that all image pairs used in our experiments (CT, T1-rec and T2-rec) were first registered by the conventional multi-resolution MI based registration method and were then examined by an experienced consultant radiologist to ensure that the final alignments are correct and acceptable. This procedure was employed for a better presentation of the probing results and also for further facilitating the experiments that will be described in Section 4.2 .

Figs. $22 \mathrm{a}$ and $2 \mathrm{~d}$ respectively plot the translational probes for registering the low resolution (Level 3) testing image pairs from three datasets for MI-I and MI-DI. At the original image resolution (Level 0), Figs. 20 and 20 plot the translational probes and Figs. $2 \mathrm{k}$ and $2 \mathrm{f}$ plot the rotational probes based on MI$\mathrm{I}$ and MI-DI respectively. As observed in Figs. $2 \mathrm{a}$ and $2 \mathrm{~b}$, for the translational probes of MI-I at different image resolutions, it would occur obvious local maxima when the misalignment of two images is relatively large. On the contrary, Figs. $2 \mathrm{~d}$ and $2 \mathrm{p}$ suggest that the shape of the probing curves based on MI-DI is improved and the capture ranges of MI-DI can be relative longer than those of MI-I. This is because, with the proposed distance-intensity attribute, regions with homogenous intensities (including anatomical structures and background regions) can provide varying information related to the distance of a voxel to its closest object boundary. Therefore, when the misalignment increases, the MI-DI values would keep decreasing. With this finding, it would be expected that the optimization procedure for registration will be benefited and the registration robustness can be increased. On the other hand, for the rotational probes, the capture ranges of MI-I and MI-DI are comparable (see Figs. 2r and 2f).

T1 - T2 (3D - 3D) Registration. Three pairs of clinical T1-rec and T2-rec image volumes - datasets \#4, \#5 and \#6 - obtained from RIRE were used for 


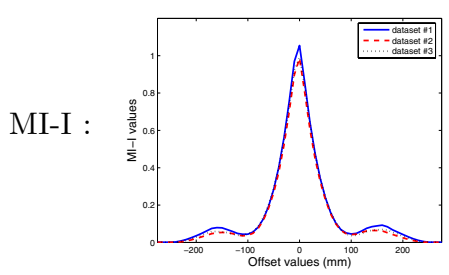

(a)

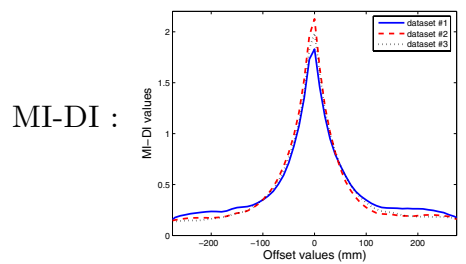

(d)

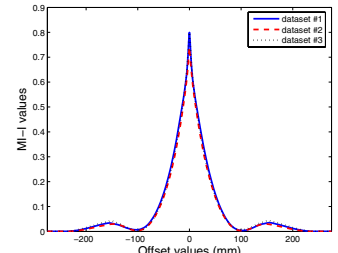

(b)

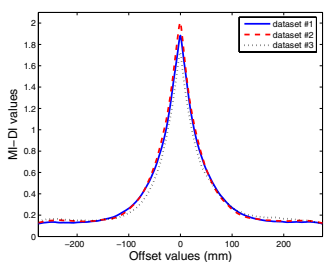

(e)

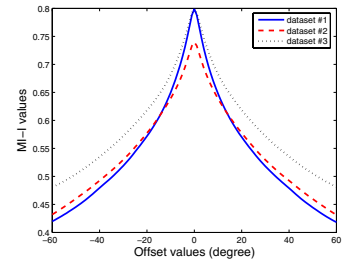

(c)

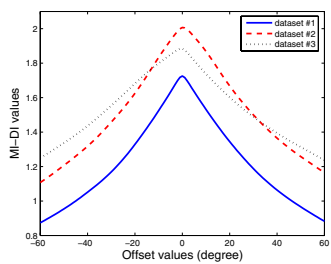

(f)

Fig. 2. Probing curves for $3 \mathrm{D}-3 \mathrm{D}$ registration on three CT and T1-rec datasets (\#1, \#2 and \#3). Translational probes for registering the low resolution (Level 3) image pairs: (a) MI-I and (d) MI-DI. Translational probes for registering the original resolution (Level 0) image pairs: (b) MI-I and (e) MI-DI. Rotational probes for registering the original resolution image pairs: (c) MI-I and (f) MI-DI.

the experiments. The results of translational probes are shown in Figs. 3 a (MII) and $3 \mathrm{~d}(\mathrm{MI}-\mathrm{DI})$ for the low resolution (Level 3) registration and in Figs. $3 \mathrm{~b}$ (MI-I) and 3e (MI-DI) for the original resolution (Level 0) registration. Figs. 3. and 3f respectively plot the rotational probes based on MI-I and MI-DI for the original resolution (Level 0). Similar results of the capture ranges are obtained as compared with $\mathrm{CT}-\mathrm{T} 1$ registrations.

\subsection{Performance Comparisons on Registration Robustness}

A series of randomized experiments have been designed to study the registration robustness of the proposed MI-DI based method and the conventional MI-I based method. The testing image pairs were datasets \#1 (CT - T1) and \#6 (T1 - T2). The experiments took 100 tests on each testing image pair for either method. At each trial, the pre-obtained ground truth registration (see Section 4.1) was perturbed by 6 uniformly distributed random offsets for all translational and rotational axes. The perturbed registration was then treated as the starting alignment. The random offsets for $\mathrm{X}$ and $\mathrm{Y}$ axes were drawn between [-150, 150] $\mathrm{mm}$, while those for $\mathrm{Z}$ axis and each rotational axis were respectively drawn between $[-70,70] \mathrm{mm}$ and $[-20,20]$ degrees. (Note that for either testing dataset the same set of randomized starting alignments was used for both methods for a fair comparison.) 


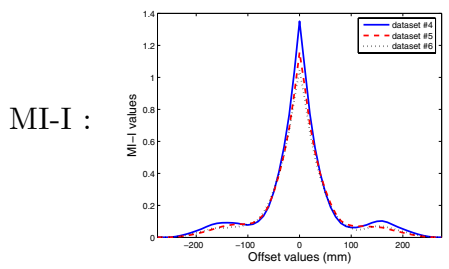

(a)

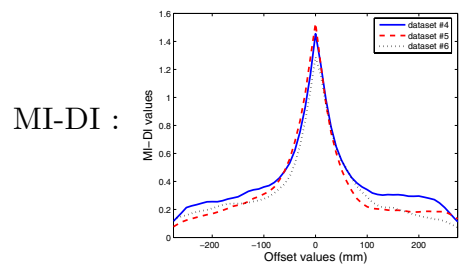

(d)

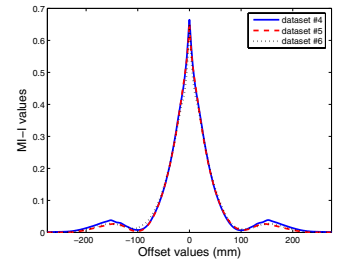

(b)

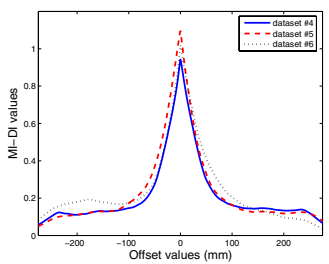

(e)

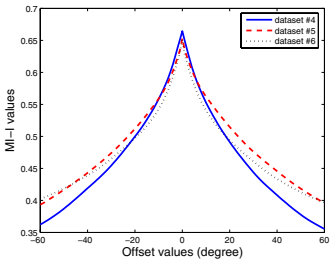

(c)

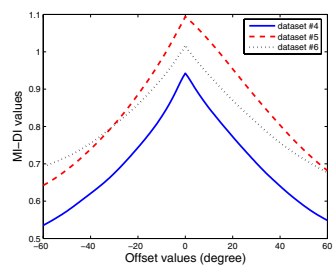

(f)

Fig. 3. Probing curves for 3D - 3D registration on 3 T1-rec and T2-rec datasets (\#4, \#5 and \#6). Translational probes for registering the low resolution (Level 3) image pairs: (a) MI-I and (d) MI-DI. Translational probes for registering the original resolution (Level 0) image pairs: (b) MI-I and (e) MI-DI. Rotational probes for registering the original resolution image pairs: (c) MI-I and (f) MI-DI.

To evaluate each derived registration with respect to the ground truth registration, the translational error (which was the root-sum-square of the differences for three translational axes) and the rotational error (which was the real part of a quaternion) were computed. In our experiments, the threshold vector for assessing registration success was set to $\left(2 \mathrm{~mm}, 2^{\circ}\right)$, because registration errors below $2 \mathrm{~mm}$ and $2^{\circ}$ are generally acceptable by experienced clinicians 1213 .

The success rates of the MI-I based method and the MI-DI based method for datasets \#1 and \#6 are listed in Table 1. It is suggested that the MI-DI based method (Column MI-DI) has higher success rates as compared with the MI-I based method (Column MI-I) for both datasets. Based on these experiments, we also observed that the majority of failed cases for the MI-DI based method had about $180^{\circ}$ misalignment for one rotational axis, while registration errors for other axes were quite small. (It is meant that, after registration, the brain in

Table 1. The success rates with the MI-I based method and the MI-DI based method for datasets \#1(CT - T1) and \#6 (T1 - T2)

\begin{tabular}{|c|c|c|}
\hline \multirow{2}{*}{$\begin{array}{c}\text { Testing } \\
\text { dataset }\end{array}$} & \multicolumn{2}{|c|}{ Success rate } \\
\cline { 2 - 3 } & MI-I & MI-DI \\
\hline$\# 1(\mathrm{CT}-\mathrm{T} 1)$ & $66 \%$ & $80 \%$ \\
\hline$\# 6(\mathrm{~T} 1-\mathrm{T} 2)$ & $68 \%$ & $85 \%$ \\
\hline
\end{tabular}


the floating image was inverted along a rotational axis.) Oppositely, for the MII based method, most of the failed cases had large translational and rotational misalignments simultaneously. This observation somehow implies that, along the translational axes, the capture ranges of MI-DI are longer than those of MI-I.

\section{Conclusion}

To conclude, this paper has designed a new one-element voxel attribute, namely distance-intensity (DI), for registration tasks. In an image at a global level, for each voxel, the DI feature encodes spatial information about the distance of the voxel to its closest object boundary, as well as the original intensity information.

The DI map of an image can be computed without image segmentations. To compute the DI map, we have demonstrated how to apply a Poisson process on a vector field combining both gradient and distance-gradient. Then, mutual information (MI) has been adopted as a similarity measure on the DI attribute space and a multi-resolution registration method has been proposed for aligning multi-modal images.

The experimental results on clinical 3D CT, MR-T1 and MR-T2 datasets have indicated that the proposed method has relatively longer capture ranges than the conventional MI-based method at different image resolutions. Moreover, a series of randomized experiments on precisely registered clinical image pairs have demonstrated that the success rates of our method are higher than those of the conventional MI-based method.

\section{Acknowledgements}

The authors would like to acknowledge the support from the Hong Kong Research Grants Council (HK RGC) under grant (HKUST6155/03E).

\section{References}

1. Maes, F., Collignon, A., et al.: Multimodality Image Registration by Maximization of Mutual Information. IEEE Trans. Med. Img. 16 (1997) 187-198

2. Wells, W., Viola, P., et al.: Multi-Modal Volume Registration by Maximization of Mutual Information. Medical Image Analysis 1 (1996) 35-51

3. Penney, G., Weese, J., et al.: A Comparison of Similarity Measures for Use in 2D-3D Medical Image Registration. IEEE Trans. Med. Img. 17 (1998) 586-595

4. Pluim, J., et al.: Image Registration by Maximization of Combined Mutual Information and Gradient Information. IEEE Trans. Med. Img. 19 (2000) 809-814

5. Pluim, J., Maintz, J., Viergever, M.: Mutual-Information-Based Registration of Medical Images: A Survey. IEEE Trans. Med. Img. 22 (2003) 986-1004

6. Rueckert, D., Clarkson, M., et al.: Non-rigid registration using higher-order mutual information. In: Proc. SPIE, Medical Imaging: Image Processing. Volume 3979. (2000) 438-447

7. Butz, T., Thiran, J.P.: Affine registration with feature space mutual information. In: MICCAI. (2001) 549-556 
8. Zwillinger, D.: Handbook of Differential Equations, 3rd ed. Boston, MA: Academic Press (1997)

9. Cover, T., et al.: Elements of Information Theory. John Wiley \& Sons, Inc. (1991)

10. Bishop, C.: Neural Networks for Pattern Recognition. Oxford U. Press (1995)

11. Press, W., Teukolsky, S., et al.: Numerical Recipes in C, 2nd Edition. Cambridge University Press (1992)

12. Hajnal, J.V., et al.: Medical Image Registration. CRC Press LLC (2001)

13. Zhu, Y., Cochoff, S.: Likelihood Maximization Approach to Image Registration. IEEE Trans. Image Processing 11 (2002) 1417-1426 